\title{
The Dynamic Modeling of Multiple Pairs of Spur Gears in Mesh, Including Friction and Geometrical Errors
}

\author{
Shengxiang Jia, Ian Howard, and Jiande Wang \\ School of Engineering, Curtin University of Technology, Bentley, Western Australia, Australia
}

This article presents a dynamic model of three shafts and two pair of gears in mesh, with 26 degrees of freedom, including the effects of variable tooth stiffness, pitch and profile errors, friction, and a localized tooth crack on one of the gears. The article also details how geometrical errors in teeth can be included in a model. The model incorporates the effects of variations in torsional mesh stiffness in gear teeth by using a common formula to describe stiffness that occurs as the gears mesh together. The comparison between the presence and absence of geometrical errors in teeth was made by using Matlab and Simulink models, which were developed from the equations of motion. The effects of pitch and profile errors on the resultant input pinion angular velocity coherent-signal of the input pinion's average are discussed by investigating some of the common diagnostic functions and changes to the frequency spectra results.

Keywords Gear, Geometrical errors, Mesh stiffness, Pitch and profile error, Simulation, Tooth crack

To improve the current generation of diagnostic techniques, many researchers are actively developing advanced dynamic models of gear vibrations to ascertain the effects of differing types of gear-train damage on the resultant gear-case vibration. One of the more recently popular modeling approaches is to use the coupled torsional and transverse motions of the shafts, along with the changes in bending stiffness that occurs as the teeth rotate through the mesh point (Du, 1997; Ozguven and Houser, 1988). The model described in this article is based on the one developed by Du (1997) and subsequently modified by Howard

Received 30 March 2003; accepted 10 June 2003.

Address correspondence to Ian Howard, Curtin University of Technology, School of Engineering, Bentley, WA 6102, Australia. E-mail: I.Howard@exchange.curtin.edu.au and colleagues (2001a, 2001b). A previous paper detailed a dynamic model of multiple pairs of gears in mesh, including friction (Howard et al., 2001b; Rebbechi et al., 1996), and it is that which has been extended here.

This article specifies how geometrical errors in teeth can be included in the model and explores the effects of localized tooth damage on the resultant vibration in the gear case. The model incorporates the effects of variations in torsional mesh stiffness in gear teeth by using a common formula for gear-mesh stiffness that occurs as the gears mesh together (Jia et al., 2001). The comparison between the conditions of presence and absence of geometrical errors in teeth was obtained by using Matlab and Simulink models, which were developed from the equations of motion. The effects of localized tooth-crack damage plus the presence of pitch and profile errors on the frequency spectrum and on the common diagnostic functions of the resulting gearbox component vibrations are also shown.

One of the difficulties with models of multiple gear pairs is recovering information about the vibrations from each shaft of interest. In this article, coherent-time, synchronous-signal averaging techniques have been used (McFadden and Smith, 1985; Forrester, 1996).

\section{MODEL AND EQUATIONS}

The model developed here is based on a two-stage reduction gearbox, in which gears 1, 2, 3, and 4 have 23, 45, 25, and 47 teeth, respectively. The overall gearbox reduction ratio is given as $(45 * 47) /(23 * 25)$, which is equivalent to a reduction ratio of 3.678. There are a total of 26 degrees of freedom in the model; a schematic diagram of the model is shown in Figure 1.

The major assumptions on which the dynamic model is based are as follows:

1. Resonances of the gearcase are neglected.

2. Shaft mass and inertia are lumped at the bearings or the gears.

3. Shaft transverse resonances are neglected. 


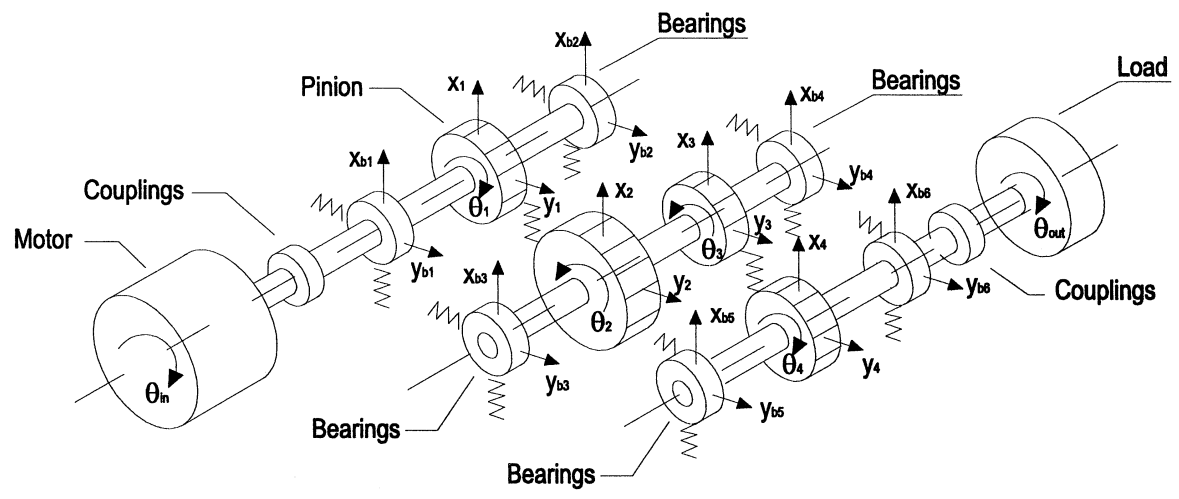

FIGURE 1

Diagram of the dynamic model of two pair of gears in mesh, with 26 degrees of freedom. The vertical direction $\left(x_{i}\right)$ is aligned with the pressure line of the gear mesh.

4. Input and output shaft torsional stiffness is ignored (flexiblecoupling torsional stiffness is very low).

5. Gear-tooth profiles are without runout errors.

6. Static transmission error effects are very much smaller than the dynamic transmission error effects, so they can be neglected.

The coupling between the torsional and transverse motions of the gears and shafts has been developed as shown in Figure 2 (Howard et al., 2001a), where the linear-translation tooth stiffness along the line of contact, $k_{\mathrm{mb}}$, can be calculated by

$$
K_{\mathrm{mb}}=\frac{K_{m}}{r_{p}^{2}}
$$

where $k_{m}$ represents the torsional mesh stiffness and $r_{p}$ denotes the radius of the pinion base circle.

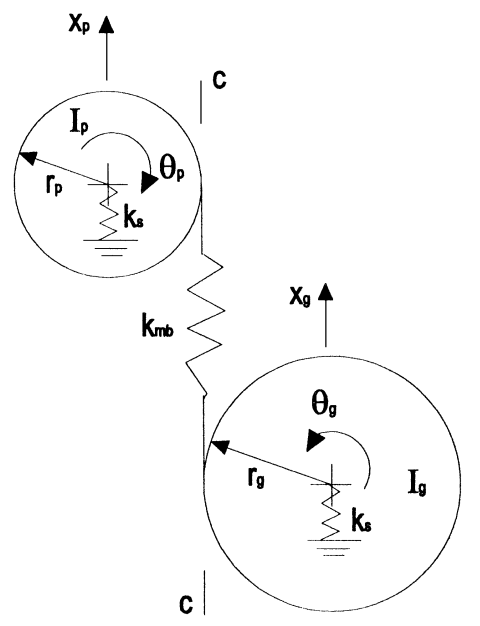

FIGURE 2

Coupling between the torsional and transverse motions of the gears and shafts. $r_{p}$, base circle radius of input pinion; $r_{g}$, base circle radius of output gear; $k_{\mathrm{mb}}$, linear-translation tooth stiffness along line of contact; c-c, line of contact.
The geometrical errors in the tooth profiles are simulated here as a forcing function input into the model and are represented by a sum of three harmonic terms, as shown in Equation 2 (Mark, 1979; Randall, 1982):

$$
e_{\mathrm{pf}}=a_{1} \sin \left(\theta_{t}\right)+b_{1} \sin \left(2 \theta_{t}\right)+c_{1} \sin \left(3 \theta_{t}\right)
$$

where $\theta_{t}$ represents the tooth meshing angle of the gear. The profile error of one couple of meshing teeth was defined as the geometrical sum of the profile geometry errors of each of the two meshing teeth because the two geometrical errors contribute to the gear vibration together.

The pitch errors were simulated as a combination of three harmonic terms, as shown in Equation (3):

$$
e_{\mathrm{pt}}=a_{2} \sin (\theta)+b_{2} \sin (2 \theta)+c_{2} \sin (3 \theta)
$$

where $\theta$ is the rotation angle of the gear.

The profile and pitch errors used in the model are shown in Figures 3 and 4 for the first and second pair of gears in mesh, respectively. A maximum geometrical error of $10 \mu \mathrm{m}$ was obtained from the combined pitch and profile deviations, as illustrated. Various amplitudes of combined geometrical error were used in the investigation, and the simulation results below compare the effects of 10 and $20 \mu \mathrm{m}$ maximum geometrical error, respectively.

The resulting equations of motion describing the coupled torsional and transverse model are given in Equations 4 through 29:

$$
\begin{aligned}
& I_{\mathrm{in}} \ddot{\theta}_{\mathrm{in}}+q_{c}\left(\dot{\theta}_{\mathrm{in}}-\dot{\theta}_{1}\right)+k_{c}\left(\theta_{\mathrm{in}}-\theta_{1}\right)=T_{\mathrm{in}}, \\
& I_{1} \ddot{\theta}_{1}+q_{c}\left(\dot{\theta}_{1}-\dot{\theta}_{\mathrm{in}}\right)+r_{1} q_{12}\left(r_{1} \dot{\theta}_{1}-r_{2} \dot{\theta}_{2}-\dot{x}_{1}+\dot{x}_{2}\right) \\
& \quad+k_{c}\left(\theta_{1}-\theta_{\mathrm{in}}\right)+r_{1} k_{12}\left(r_{1} \theta_{1}-r_{2} \theta_{2}-x_{1}+x_{2}\right) \\
& \quad-r_{1} k_{12}\left(e_{12}+e_{1 \mathrm{pt}}+e_{2 \mathrm{pt}}\right)+F_{f 12} r_{f 1}=0, \\
& \quad I_{2} \ddot{\theta}_{2}+r_{2} q_{12}\left(r_{2} \dot{\theta}_{2}-r_{1} \dot{\theta}_{1}-\dot{x}_{2}+\dot{x}_{1}\right)+k_{\mathrm{st}}\left(\theta_{2}-\theta_{3}\right) \\
& \quad+r_{2} k_{12}\left(r_{2} \theta_{2}-r_{1} \theta_{1}-x_{2}+x_{1}\right) \\
& \quad+r_{2} k_{12}\left(e_{12}+e_{1 \mathrm{pt}}+e_{2 \mathrm{pt}}\right)-F_{f 12} r_{f 2}=0,
\end{aligned}
$$



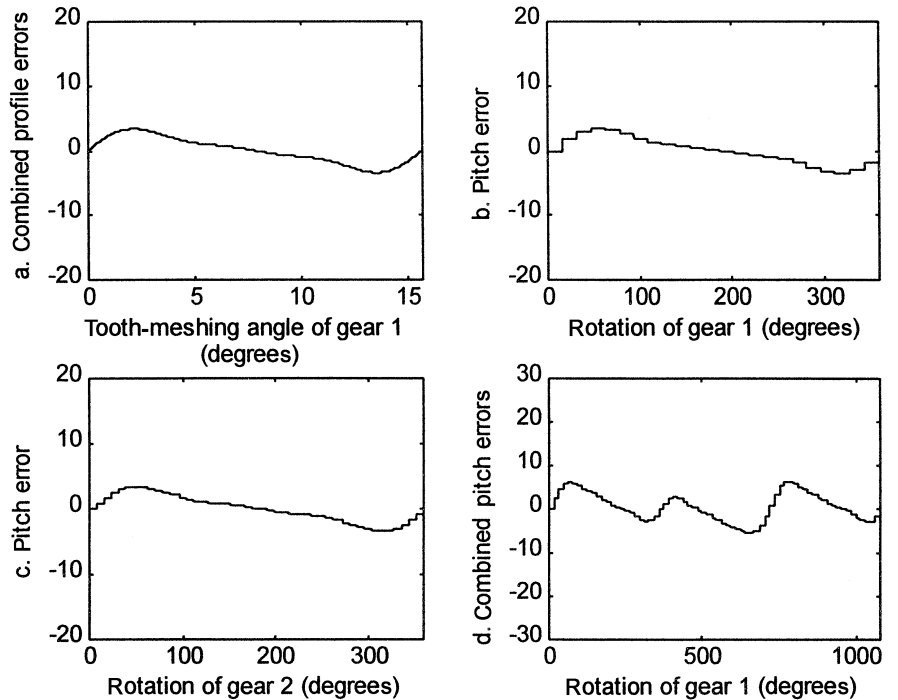

FIGURE 3

Profile and pitch errors of the first gear mesh. (a) Combined profile errors of gears 1 and 2. (b) Pitch error of gear 1. (c) Pitch error of gear 2. (d) Combined pitch errors of gears 1 and 2 . The units of profile and pitch error are $\mu \mathrm{m}$.

$$
\begin{aligned}
& I_{3} \ddot{\theta}_{3}+r_{3} q_{34}\left(r_{3} \dot{\theta}_{3}-r_{4} \dot{\theta}_{4}+\dot{x}_{3}-\dot{x}_{4}\right)+k_{\mathrm{st}}\left(\theta_{3}-\theta_{2}\right) \\
& \quad+r_{3} k_{34}\left(r_{3} \theta_{3}-r_{4} \theta_{4}+x_{3}-x_{4}\right)-r_{3} k_{34}\left(e_{34}+e_{3 \mathrm{pt}}+e_{4 \mathrm{pt}}\right) \\
& \quad+F_{f 34} r_{f 3}=0, \\
& I_{4} \ddot{\theta}_{4}+r_{4} q_{34}\left(r_{4} \dot{\theta}_{4}-r_{3} \dot{\theta}_{3}+\dot{x}_{4}-\dot{x}_{3}\right)+k_{c}\left(\theta_{4}-\theta_{\mathrm{out}}\right) \\
& \quad+r_{4} k_{34}\left(r_{4} \theta_{4}-r_{3} \theta_{3}+x_{4}-x_{3}\right)+r_{4} k_{34}\left(e_{34}+e_{3 \mathrm{pt}}+e_{4 \mathrm{pt}}\right) \\
& \quad-F_{f 34} r_{f 4}=0,
\end{aligned}
$$
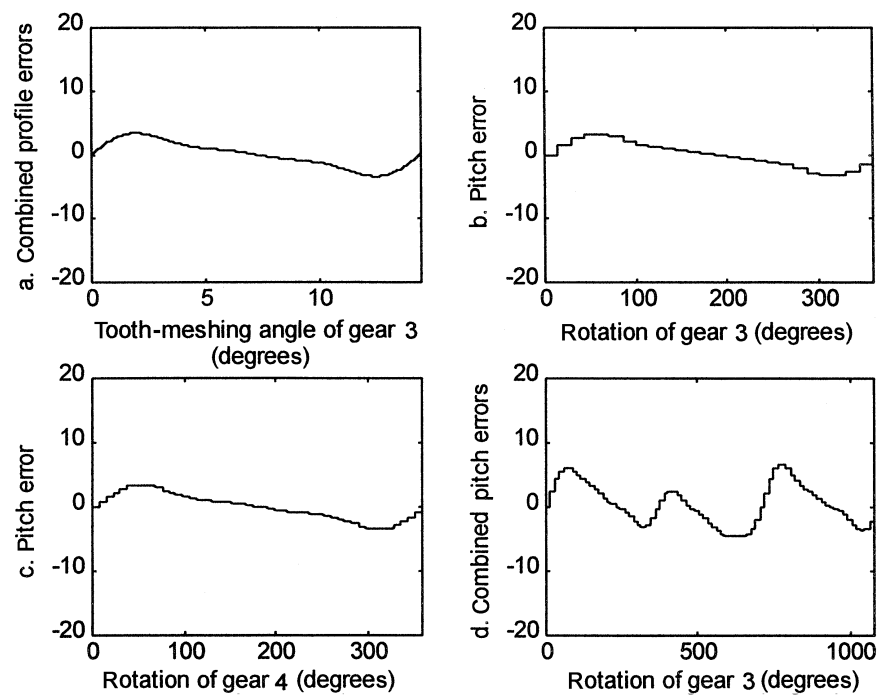

FIGURE 4

Profile and pitch errors of the second gear mesh. (a) Combined profile errors of gears 3 and 4. (b) Pitch error of gear 3. (c)

Pitch error of gear 4. (d) Combined pitch errors of gears 3 and 4. The units of profile and pitch error are $\mu \mathrm{m}$.

$$
\begin{aligned}
& I_{L} \ddot{\theta}_{\text {out }}+q_{c}\left(\dot{\theta}_{\text {out }}-\dot{\theta}_{4}\right)+k_{c}\left(\theta_{\text {out }}-\theta_{4}\right)=T_{\text {Load }}, \\
& m_{1} \ddot{x}_{1}+k_{s}\left(x_{1}-x_{b 1}\right)+k_{s}\left(x_{1}-x_{b 2}\right) \\
& +k_{12}\left(x_{1}-x_{2}-r_{1} \theta_{1}+r_{2} \theta_{2}\right)-k_{12}\left(e_{12}+e_{1 \mathrm{pt}}+e_{2 \mathrm{pt}}\right) \\
& +q_{12}\left(\dot{x}_{1}-\dot{x}_{2}-r_{1} \dot{\theta}_{1}+r_{2} \dot{\theta}_{2}\right)=0 \text {, } \\
& m_{2} \ddot{x}_{2}+k_{s}\left(x_{2}-x_{b 3}\right)+k_{s}\left(x_{2}-x_{3}\right) \\
& +k_{12}\left(x_{2}-x_{1}-r_{2} \theta_{2}+r_{1} \theta_{1}\right)+k_{12}\left(e_{12}+e_{1 \mathrm{pt}}+e_{2 \mathrm{pt}}\right) \\
& +q_{12}\left(\dot{x}_{2}-\dot{x}_{1}-r_{2} \dot{\theta}_{2}+r_{1} \dot{\theta}_{1}\right)=0 \text {, } \\
& m_{3} \ddot{x}_{3}+k_{s}\left(x_{3}-x_{2}\right)+k_{s}\left(x_{3}-x_{b 4}\right) \\
& +k_{34}\left(x_{3}-x_{4}+r_{3} \theta_{3}-r_{4} \theta_{4}\right)-k_{34}\left(e_{34}+e_{3 \mathrm{pt}}+e_{4 \mathrm{pt}}\right) \\
& +q_{34}\left(\dot{x}_{3}-\dot{x}_{4}+r_{3} \dot{\theta}_{3}-r_{4} \dot{\theta}_{4}\right)=0 \text {, } \\
& m_{4} \ddot{x}_{4}+k_{s}\left(x_{4}-x_{b 5}\right)+k_{s}\left(x_{4}-x_{b 6}\right) \\
& +k_{34}\left(x_{4}-x_{3}+r_{4} \theta_{4}-r_{3} \theta_{3}\right)+k_{34}\left(e_{34}+e_{3 \mathrm{pt}}+e_{4 \mathrm{pt}}\right) \\
& +q_{34}\left(\dot{x}_{4}-\dot{x}_{3}+r_{4} \dot{\theta}_{4}-r_{3} \dot{\theta}_{3}\right)=0 \text {, } \\
& m_{1} \ddot{y}_{1}+k_{s}\left(y_{1}-y_{b 1}\right)+k_{s}\left(y_{1}-y_{b 2}\right)-F_{f 12}=0 \text {, } \\
& m_{2} \ddot{y}_{2}+k_{s}\left(y_{2}-y_{b 3}\right)+k_{s}\left(y_{2}-y_{3}\right)+F_{f 12}=0 \text {, } \\
& m_{3} \ddot{y}_{3}+k_{s}\left(y_{3}-y_{2}\right)+k_{s}\left(y_{3}-y_{b 4}\right)+F_{f 34}=0 \text {, } \\
& m_{4} \ddot{y}_{4}+k_{s}\left(y_{4}-y_{b 5}\right)+k_{s}\left(y_{4}-y_{b 6}\right)-F_{f 34}=0 \text {, } \\
& m_{b} \ddot{x}_{b 1}+q_{b} \dot{x}_{b 1}+k_{b} x_{b 1}+k_{s}\left(x_{b 1}-x_{1}\right)=0 \text {, } \\
& m_{b} \ddot{x}_{b 2}+q_{b} \dot{x}_{b 2}+k_{b} x_{b 2}+k_{s}\left(x_{b 2}-x_{1}\right)=0 \text {, } \\
& m_{b} \ddot{x}_{b 3}+q_{b} \dot{x}_{b 3}+k_{b} x_{b 3}+k_{s}\left(x_{b 3}-x_{2}\right)=0 \text {, } \\
& m_{b} \ddot{x}_{b 4}+q_{b} \dot{x}_{b 4}+k_{b} x_{b 4}+k_{s}\left(x_{b 4}-x_{3}\right)=0 \text {, } \\
& m_{b} \ddot{x}_{b 5}+q_{b} \dot{x}_{b 5}+k_{b} x_{b 5}+k_{s}\left(x_{b 5}-x_{4}\right)=0 \text {, } \\
& m_{b} \ddot{x}_{b 6}+q_{b} \dot{x}_{b 6}+k_{b} x_{b 6}+k_{s}\left(x_{b 6}-x_{4}\right)=0 \text {, } \\
& m_{b} \ddot{y}_{b 1}+q_{b} \dot{y}_{b 1}+k_{b} y_{b 1}+k_{s}\left(y_{b 1}-y_{1}\right)=0 \text {, } \\
& m_{b} \ddot{y}_{b 2}+q_{b} \dot{y}_{b 2}+k_{b} y_{b 2}+k_{s}\left(y_{b 2}-y_{1}\right)=0 \text {, } \\
& m_{b} \ddot{y}_{b 3}+q_{b} \dot{y}_{b 3}+k_{b} y_{b 3}+k_{s}\left(y_{b 3}-y_{2}\right)=0 \text {, } \\
& m_{b} \ddot{y}_{b 4}+q_{b} \dot{y}_{b 4}+k_{b} y_{b 4}+k_{s}\left(y_{b 4}-y_{3}\right)=0 \text {, } \\
& m_{b} \ddot{y}_{b 5}+q_{b} \dot{y}_{b 5}+k_{b} y_{b 5}+k_{s}\left(y_{b 5}-y_{4}\right)=0 \text {, }
\end{aligned}
$$

and

$$
m_{b} \ddot{y}_{b 6}+q_{b} \dot{y}_{b 6}+k_{b} y_{b 6}+k_{s}\left(y_{b 6}-y_{4}\right)=0 .
$$

\section{CONTACT FORCE, FRICTION FORCE, AND FRICTION MOMENT}

The equation for the normal contact force between gear 1 and gear 2 is given by Equation 30, and the friction force between the gears is calculated by Equation 31 (Howard et al., 2001a):

$$
\begin{aligned}
F_{12}= & k_{12}\left(x_{2}-x_{1}-r_{2} \theta_{2}+r_{1} \theta_{1}\right)+k_{12}\left(e_{12}+e_{1 \mathrm{pt}}+e_{2 \mathrm{pt}}\right) \\
& +q_{12}\left(\dot{x}_{2}-\dot{x}_{1}-r_{2} \dot{\theta}_{2}+r_{1} \dot{\theta}_{1}\right)
\end{aligned}
$$

and

$$
F_{f 12}=\mu F_{12} .
$$

Likewise, the normal contact force between gear 3 and gear 4 is given by Equation 32, and the value of the friction force 
between the two gears is calculated by Equation 33:

$$
\begin{aligned}
F_{34}= & k_{34}\left(x_{3}-x_{4}+r_{3} \theta_{3}-r_{4} \theta_{4}\right)-k_{34}\left(e_{34}+e_{3 \mathrm{pt}}+e_{4 \mathrm{pt}}\right) \\
& +q_{34}\left(\dot{x}_{3}-\dot{x}_{4}+r_{3} \dot{\theta}_{3}-r_{4} \dot{\theta}_{4}\right) \\
F_{f 34}= & \mu F_{34} .
\end{aligned}
$$

The above equations require the torsional mesh stiffness, the ratio of the contact force between the teeth, and the dynamic friction coefficient (Rebbechi, 1996) to be known at every position of the gears throughout the simulation. Figure 5 shows the parameters used for the results shown in this article for steel gears of module 6 with teeth numbers $23,45,25$, and 47 . In Equations 5 through $8, r_{f 1}, r_{f 2}, r_{f 3}$, and $r_{f 4}$ denote the friction moment arms on gears 1 through 4 , respectively, as obtained by geometrical analysis (Howard et al., 2001).

\section{NUMERIC SIMULATION}

The dynamic simulation of the differential equations of motion was achieved using Simulink software. After the steadystate rotational speed of the input shaft had been achieved, 100

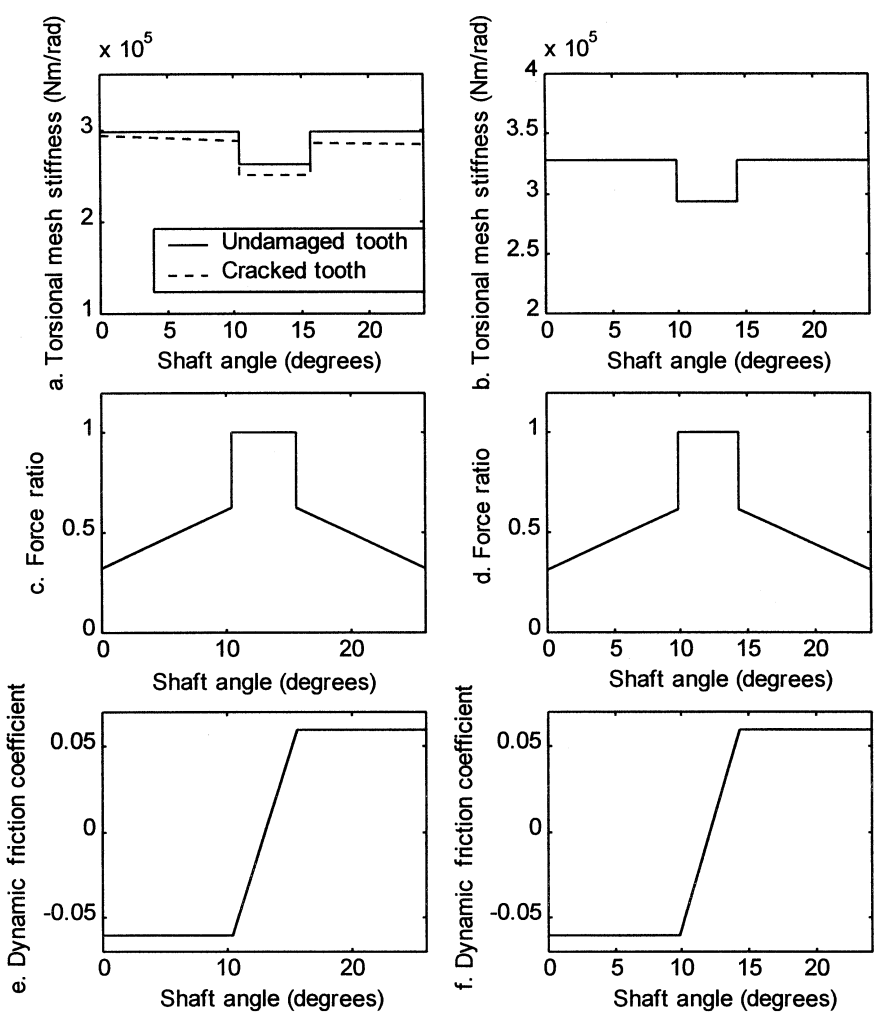

FIGURE 5

Parameters used throughout the gear simulation. (a) Torsional mesh stiffness between gears 1 and 2. (b) Torsional mesh stiffness between gears 3 and 4. (c) Load-sharing ratio between gears 1 and 2. (d) Load-sharing ratio between gears 3 and 4. (e)

Dynamic friction coefficient between gears 1 and 2. (f)

Dynamic friction coefficient between gears 3 and 4 .
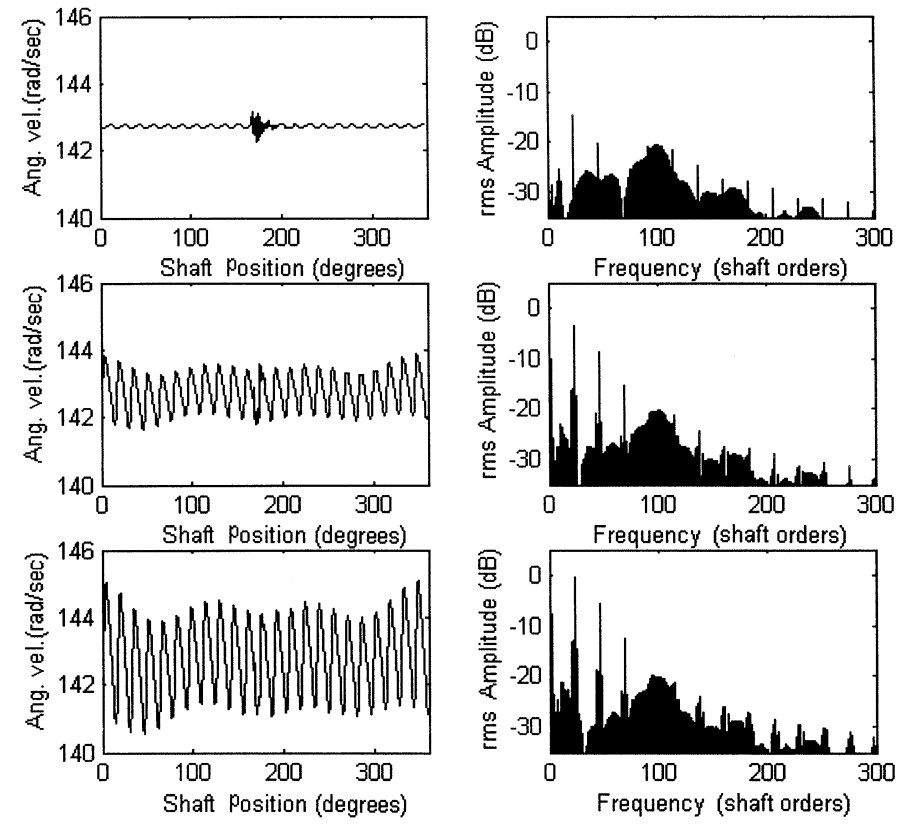

FIGURE 6

The input shaft angular velocity $\dot{\theta}_{1}$ after 50 averages, with geometrical error magnitudes of 0,10 , and $20 \mu \mathrm{m}$, respectively, and the corresponding rms amplitude spectra.

revolutions of the input shaft $(5 \mathrm{sec})$ were simulated for subsequent analysis. Coherent synchronous signal-averaging techniques were used to recover the vibration information relating to the shafts of interest (Forrester, 1996; McFadden, 1986; McFadden and Smith, 1985). The simulation was used to illustrate the effects of the geometrical errors and a single tooth crack on the resultant vibration. One of the 23 teeth on the input shaft (tooth number 11) had a simulated 5-mm fillet crack at the root of a tooth, as represented by a change in torsional mesh stiffness, which is shown in Figure 5a.

The resultant signal average obtained using 50 averages of the input pinion velocity $\dot{\theta}_{1}$ and the corresponding (Root Mean Square) amplitude spectra are shown in Figure 6, with various amplitudes of geometrical error.

The presence of the 5-mm fillet crack can be readily observed in the input pinion vibration from the signal average. Its position corresponds to tooth number 11, as expected. As the geometrical error is introduced into the model, it becomes an additional forcing input to the system, and the effect of the tooth crack is not as apparent. With a tooth-profile error of $20 \mu \mathrm{m}$, the crack is barely seen in the signal average.

The tooth-profile error was modeled as a periodic function that is periodic with the tooth spacing, as shown in Figures 3 and 4. It can be observed in Figure 6 that the increase in the geometrical error magnitude places more spectral energy into the tooth-mesh harmonics. Also observed in the results was the addition of sidebands around the gear-mesh harmonics. This also was expected, as the profile error for each tooth was modeled as 

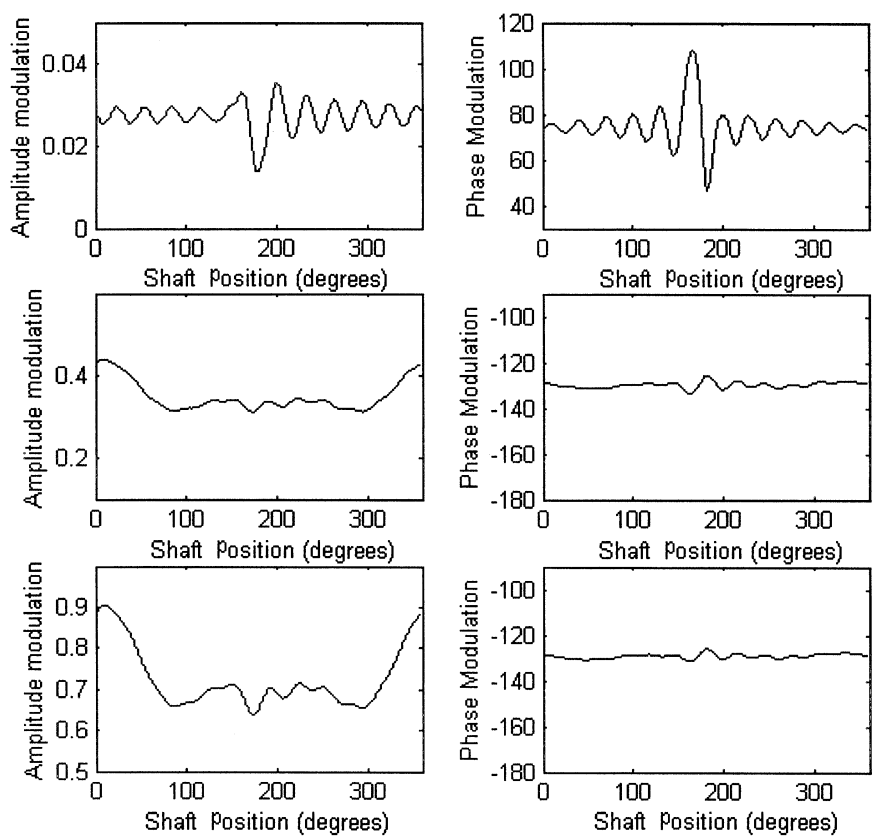

\section{FIGURE 7}

Amplitude and phase modulation of the input pinion's angular velocity for increasing amplitudes of geometrical error.

Maximum combined tooth error, $0 \mu \mathrm{m}, 10 \mu \mathrm{m}$, and $20 \mu \mathrm{m}$.

the sum of three harmonic terms, which is illustrated in Equation 2. The amplitude and phase modulation of the fundamental mesh frequency of the input pinion's angular velocity are shown in Figure 7 for increasing magnitudes of geometrical error. It can be observed in the results that the geometrical errors cloud the modulation changes caused by the tooth crack. For geometrical errors of just $10 \mu \mathrm{m}$, the modulation changes induced by the 5-mm tooth crack appear to be obscured. Other common diagnostic functions such as the kurtosis of the residual signal and narrow band envelope (Forrester, 1996; McFadden, 1986) also appear to be ineffective in detecting the tooth crack.

\section{DISCUSSION AND CONCLUSION}

The simulation results shown in this paper indicate that pitch and profile errors of 10 microns have a significant impact on the gearbox vibration. The geometrical errors are observed to provide additional energy into the tooth mesh harmonics and associated sidebands, clouding the modulation changes caused by localised tooth damage such as a tooth crack. A tooth crack of $5 \mathrm{~mm}$ length was seen to be difficult to diagnose with geometrical pitch and profile errors of 10 microns.

\section{NOMENCLATURE}

$F_{12} \quad$ Tooth friction force between gear 1 and gear 2

$F_{34} \quad$ Tooth friction force between gear 3 and gear 4

$I_{L} \quad$ Mass moment of inertia of the output load

$I_{\text {in }} \quad$ Mass moment of inertia of the motor
$I_{i} \quad i=1-4$, Mass moments of inertia of gears 1, 2, 3, and 4

$k_{b} \quad$ Radial stiffness of the bearing and support

$k_{c} \quad$ Torsional stiffness of the flexible coupling

$k_{s} \quad$ Shaft transverse stiffness

$k_{s t} \quad$ Shaft torsional stiffness

$k_{12}$. Bending tooth stiffness between gear 1 and gear 2

$k_{34} \quad$ Bending tooth stiffness between gear 3, and gear 4

$m_{b} \quad$ Mass of the bearing and part of the shaft

$m_{i} \quad i=1-4$, Masses of gears 1, 2, 3, and 4

$r_{f i} \quad i=1-4$, Friction moment arms on gear 1, 2, 3, and 4

$q_{b} \quad$ Viscous damping coefficient of bearing pinion, gear, and load

$q_{c} \quad$ Viscous damping coefficient of flexible coupling

$q_{12} \quad$ Viscous damping coefficient of gear 2 and 3 mesh

$q_{34} \quad$ Viscous damping coefficient of gear 2 and 3 mesh

$r_{i} \quad i=1-4$, Base circle radius of gears $1,2,3$, and 4

$T_{\text {in }} \quad$ Input motor torque

$T_{\text {load }} \quad$ Load torque from load

$x_{i} \quad i=1-4$, Linear vertical displacement of gears $1,2,3$, and 4

$y_{i} \quad i=1-4$, Linear level displacement of gears $1,2,3$, and 4

$x_{\mathrm{bi}} \quad i=1-6$, Linear vertical displacement of bearings 1-6

$y_{\text {bi }} \quad i=1-6$, Linear level displacement of bearings 1-6

$\theta_{i} \quad i=1-4$, Angular displacements of gears 1,2, 3, and 4

$\theta_{\text {in }} \quad$ Angular displacements of motor, load

$\theta_{\text {out }} \quad$ Angular displacements of motor, load

$\mu \quad$ Dynamic coefficient of friction

$e_{12}$ Combined profile error of gear 1 and gear 2 tooth mesh

$e_{34}$ Combined profile error of gear 3 and gear 4 tooth mesh

$e_{1 \mathrm{pt}} \quad$ Pitch error of gear 1

$e_{2 \mathrm{pt}} \quad$ Pitch error of gear 2

$e_{3 \mathrm{pt}} \quad$ Pitch error of gear 3

$e_{4 \mathrm{pt}} \quad$ Pitch error of gear 4

\section{REFERENCES}

Du, S. 1997. Dynamic Modelling and Simulation of Gear Transmission Error for Gearbox Vibration Analysis (PhD diss, University of New South Wales).

Forrester, B. D. 1996. Advanced Vibration Analysis Techniques for Fault Detection and Diagnosis in Geared Transmission Systems (PhD diss, Swinburne University of Technology).

Howard, I., Jia, S., and Wang, J. 2001a. The dynamic modeling of a spur gear in mesh, including friction and a crack. Journal of Mechanical Systems and Signal Processing 15:831-853.

Howard, I., Jia, S., and Wang, J. 2001b, September. The dynamic modeling of multiple pairs of spur gears in mesh, including friction. Proceedings of the 14th International Conference on Condition Monitoring and Diagnostic Engineering Management, 841-848. Manchester: ELSEVIER Science Ltd.

Jia, S., Howard, I., and Wang, J. 2001, November. A common formula for spur gear mesh stiffness. Proceedings of the JSME International Conference on Motion and Power Transmissions, 1-4. Fukuoka: JSME.

Mark, W. D. 1979. Analysis of the vibration excitation of a gear system. II: Tooth error representations, approximations, and 
application. Journal of the Acoustical Society of America 66:17581787.

McFadden, P. D. 1986. Detecting fatigue cracks in gears by amplitude and phase demodulation of the meshing vibration. Journal of Vibration, Acoustics, Stress and Reliability in Design 108:165-170.

McFadden, P. D., and Smith, J. D. 1985. A signal processing technique for detecting local defects in a gear from the signal average of the vibration. Proceedings of the Institution of Mechanical Engineers 199:287-292.
Ozguven, H. N., and Houser, D. R. 1988. Mathematical models used in gear dynamics: A review. Journal of Sound and Vibration 121:383411.

Randall, R. B. 1982. A new method of modeling gear faults. Journal of Mechanical Design 104:259.

Rebbechi, B., Oswald, F., and Townsend, D. 1996. Measurement of gear tooth dynamic friction. Proceedings of the Power Transmission and Gearing Conference, Paper ASME DE-88, 355-363. San Diego: ASME. 

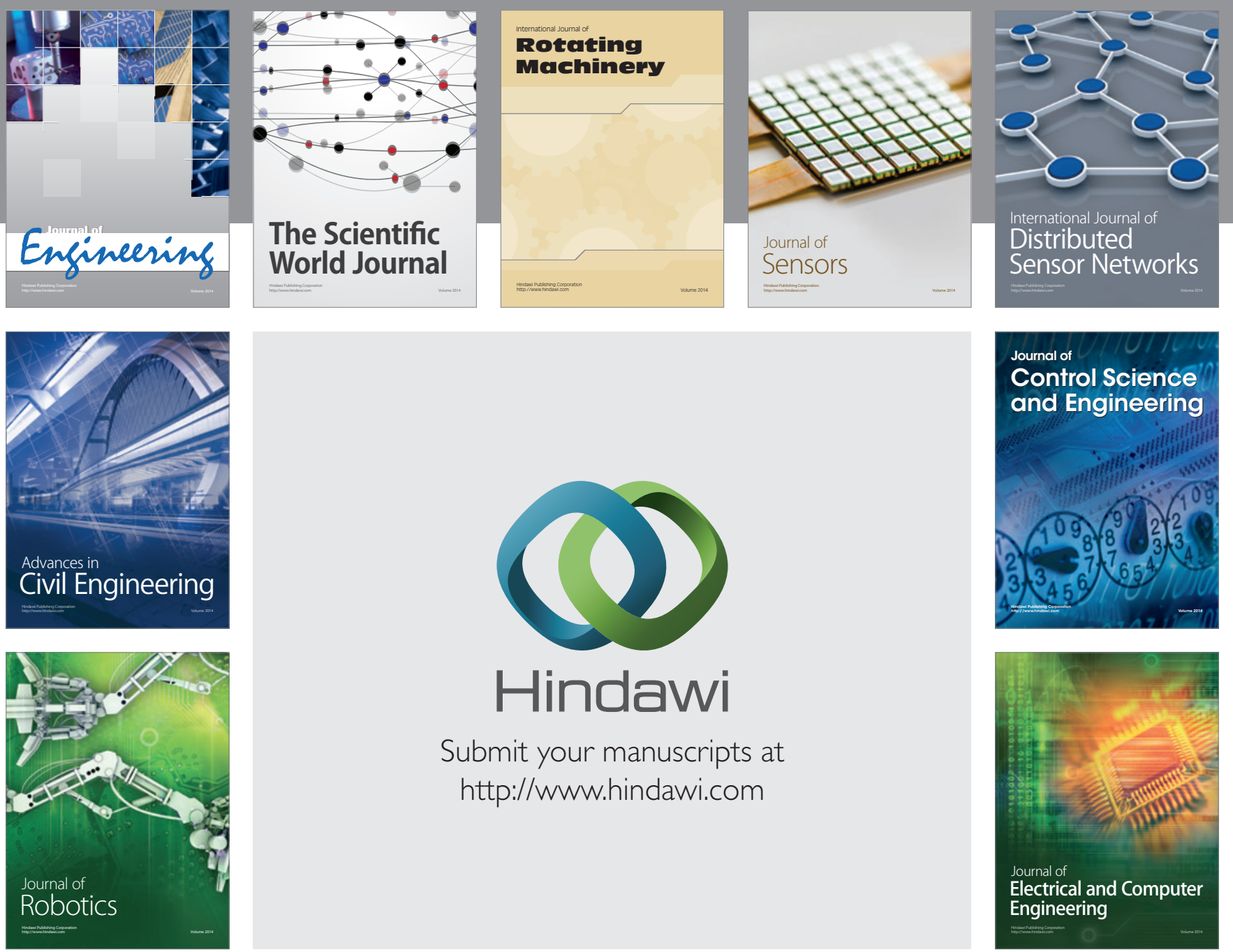

Submit your manuscripts at

http://www.hindawi.com
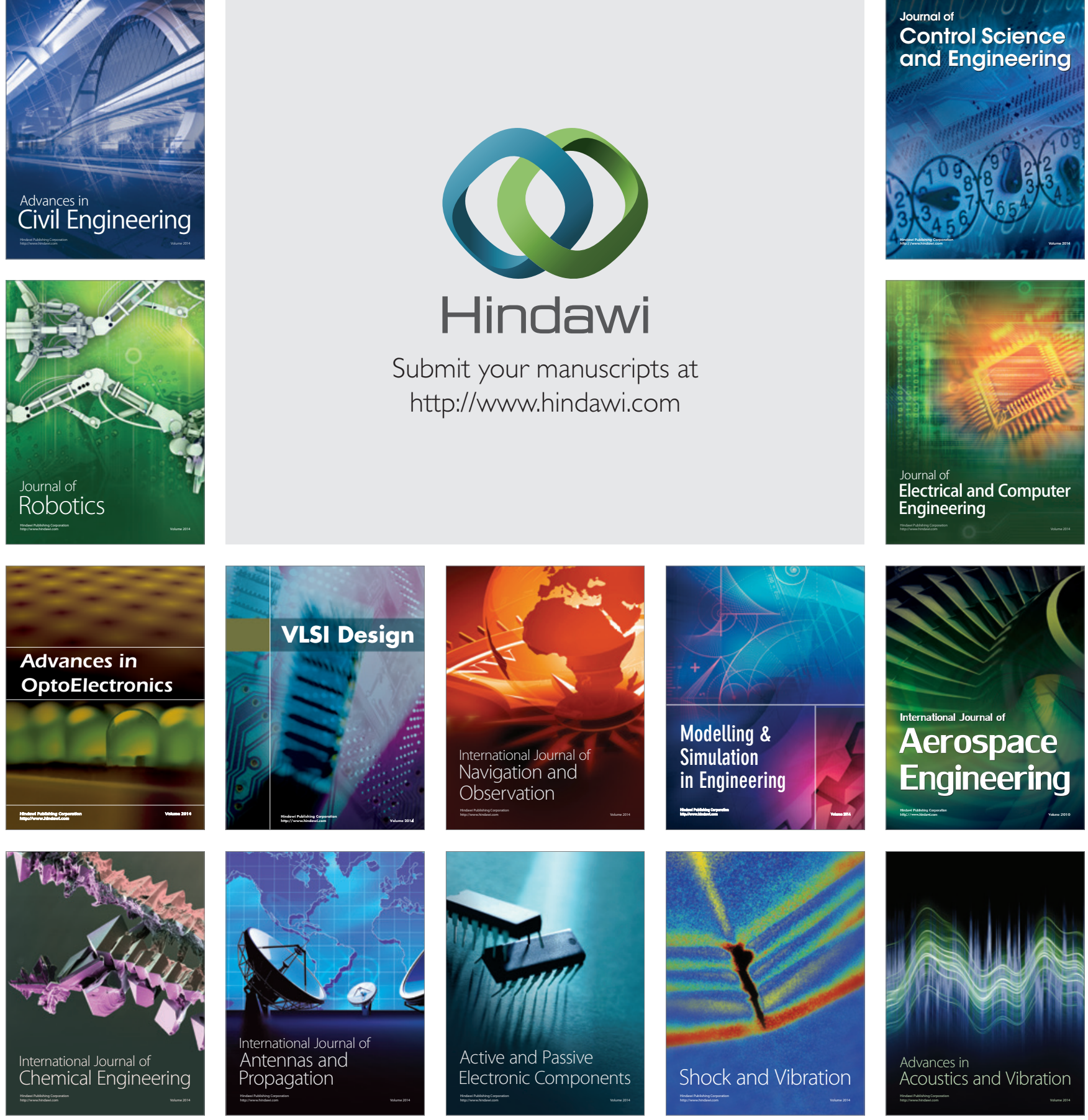